\title{
A Framework Study for Healthcare Information Systems
}

\author{
Celal Çeken \\ Computer Engineering Department, Faculty of Computer and Information Sciences, Sakarya University, Sakarya, \\ Türkiye \\ Email: celalceken@sakarya.edu.tr
}

Received July 2014

\begin{abstract}
Healthcare information systems are crucial components for better coordination of healthcare. They focus on the proper generation, transmission, storage, and retrieval of health data. It is obvious that production of accurate, relevant, and timely health information is foundation of good decision making. Rapid progress in wireless communications and embedded systems result in wireless sensor networks to be employed even in biomedical applications as well as their prominent deployment options. This study proposes a healthcare information system framework which consists of such components as; wireless sensor networks, cellular networks, a MATLAB interface, a database, and a web based monitoring interface. A case study that includes sensing, transferring, storing and web based monitoring processes of ECG signal is also introduced in the study, so that the behavior of the system developed can be tested. The results show that the framework presented here can not only be employed as a healthcare information system, but it can also be used as an infrastructure in related research activities and consequently, lots of time can be saved from creating an experimental environment.
\end{abstract}

\section{Keywords}

Healthcare Information System, Biomedical Automation, Wireless Sensor Networks, Web-Based Healthcare System Framework

\section{Introduction}

The healthcare information systems are vital for decision-making and have such functions as; data generation, compilation, analysis and synthesis, and finally communication and use. Accurate, relevant, and timely information is key to decision making and is essential for health system policy development and implementation, governance and regulation, health research, human resources development, health education and training, service delivery and financing [1] [2].

The goal of this study is to develop a healthcare information system framework which consists of several modules such as; wireless sensor networks (WSNs), cellular networks (CNs), a MATLAB interface, a database, and a web based monitoring interface.

WSN module, can also be referred as a body area network, includes sensing nodes (SNs) and an access point 
(AP). A SN is an embedded device that is capable of sensing biological signals such as; heart rate, blood pressure, oxygen saturation levels, blood glucose, brain activity, etc. from human body and can send them to the AP using its wireless interface. Well-known ZigBee protocol is employed in the WSN and this module is modeled and simulated using OPNET Modeler Software for performance evaluation. CN structure has a BS and two transceiver interfaces which are integrated into the nodes AP in WSN and Server in Data Center. CN employs GSM/GPRS protocol so that the biological data sensed can be delivered to the Data Center (DC), and modeled using OPNET Modeler as well.

The framework has also a MATLAB interface which is presently monitor the sensed data (later, it will be utilized in potential upcoming studies). Finally, the web based monitoring interface developed is responsible for revealing the sensed biological data clearly and storing it into the database. In order to test the framework, a case study that includes sensing, transferring, storing and web based monitoring processes of ECG signal is also introduced in the study.

The contributions of this study can be summarized as follows:

- A new healthcare information system framework has been designed and implemented using several components and various tools.

- Even though building a healthcare information system is quite complicated, related research studies can easily be realized by means of the framework developed, which implies that lots of time can be saved from creating an experimental environment.

- A new bridge node (AP) that receives the sensed data from SNs using ZigBee protocol and delivers to the Server in DC using GSM/GPRS, is developed.

The remainder of the paper is organized as follows: In Section 2, a brief literature search is given related to the study. Overall properties and the components of the framework introduced are presented in Section 3. Section 4 presents a case study that includes sensing, transferring, storing and web based monitoring processes of ECG signal using the framework developed. The paper is concluded with the last section providing summary about the study with final remarks.

\section{Related Works}

Several studies related to healthcare information systems can be found in the literature. In [3] [4], the authors design a test platform based on TTCN-3 standard for interoperability testing of healthcare applications. The article [5] introduces a new XML-aware compression technique for improving performance of healthcare information systems. In the study, two XML-aware compressors compress patient messages transferred between Web clients and servers. In order to manage complex medical data, a framework for interoperable healthcare information systems is proposed in [6]. In [7], a Smart Healthcare Systems Framework is proposed for conceptualizing data-driven and mobile/cloud enabled smart healthcare systems. Another healthcare information system is introduced in [8] that enables standardized exchange and homogeneous management of ECG formats.

\section{Architecture of the Framework}

The framework developed consists of several components such as; body area network or WSN, cellular networks (CN), MATLAB interfaces, a database structure, and a web based monitoring interface. Here in this section, all of the framework modules that are outlined in Figure $\mathbf{4}$ will be explained.

\subsection{Body Area Network}

WSN technology can commonly be deployed in industrial, medical, military, and environmental areas, for monitoring, tracking, data processing and decision making purposes. ZigBee is based on an IEEE 802.15 standard and is a specification for a suite of high level communication protocols used to create wireless personal/body area networks [9]. In the framework, SNs firstly senses the biological signal from the human body and then transferred it to the AP using ZigBee protocol. SNs and AP are both modeled in OPNET Modeler Software that is a powerful event-driven tool for modeling and simulating various electronic communications systems. The Node Model of the SNs built in OPNET is illustrated in Figure 1.

Sink, SourceGenerator, Network, and DLL modules in the figure are programmed using Proto-C language. Proto- $\mathrm{C}$ is a special language includes state machines, transitions between states and $\mathrm{C} / \mathrm{C}++$ language. In SourceGenerator module, for the case study presented in the next section, the ECG signal is read from the "ECG.txt" 


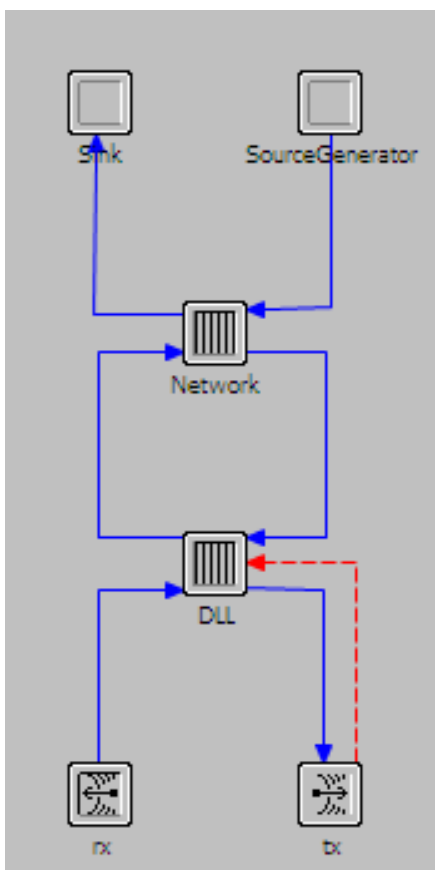

Figure 1. SN node model.

file, inserted into the ZigBee data packet and then, conveyed to the lower layers Network and DLL, which are responsible for ZigBee functionalities. Finally, physical layer (rx, tx) is in charge of providing an interface between the node module and air.

Node model of the AP is given in Figure 2. The key function of the AP is encapsulating the Protocol Data Units (PDU) of the ZigBee messages into those of the GSM/GPRS frames to be carried to the Server in Data Center. For the opposite direction, reverse translation process is supported as well. It actually behaves like a bridge between WSN and GSM network. Any sensed biological signal is transferred from SNs to the AP, and then AP delivers this data to the Data Center, and vice versa.

\subsection{Web Based Monitoring Sub-System}

The web-based monitoring interface (depicted in Figure 3) for the framework is developed by using tools such as; PHP web programming language, Apache web server, jQuery java script framework, HTML and MySQL database management system. Ajax technology is also incorporated into the user interface so that enhanced user-website interaction and lower bandwidth usage can be achieved. After the sensed biological data arrive at the Server in Data Center, it is stored in the relational database for further considerations.

The relational model of the database is illustrated in Figure 4. Related information about the employees and patients is stored in corresponding tables of the database designed. All the meta data related to the patients and the measured biological signals is stored in "records" table. The raw biological data is stored in classic files while the paths of these files are saved in "dataFile" field of the "records" table.

Only registered employees are allowed to enter the web based user interface given in Figure 3, in order to monitor the biological signals stored. Employees can apply filters and search for the desired records using left side menu, i.e. Filter Records, on the interface. After the search button is pressed, results are listed on the right side, i.e. Search Results. Any biological data in the list can be drawn using the "-->>" sign in the "Show" column.

\subsection{MATLAB Module}

In order to support any potential upcoming studies which are listed in Section 4, MATLAB interfaces are provided as well. In the case study, explained in the next section, MATLAB is employed in AP and Server nodes only for monitoring process of biological signals in real time. 


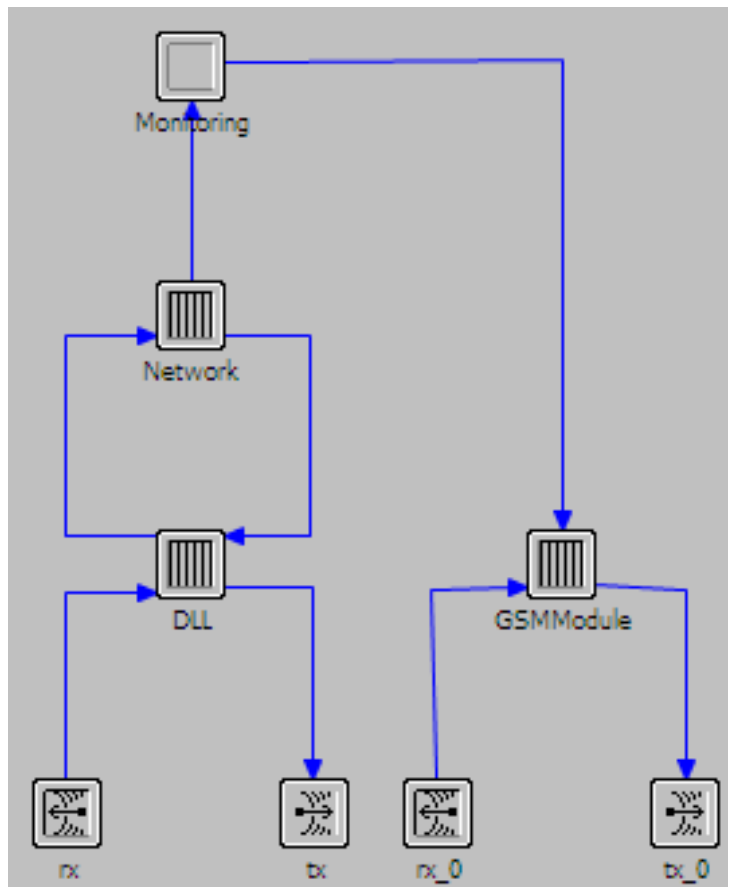

Figure 2. AP node model.

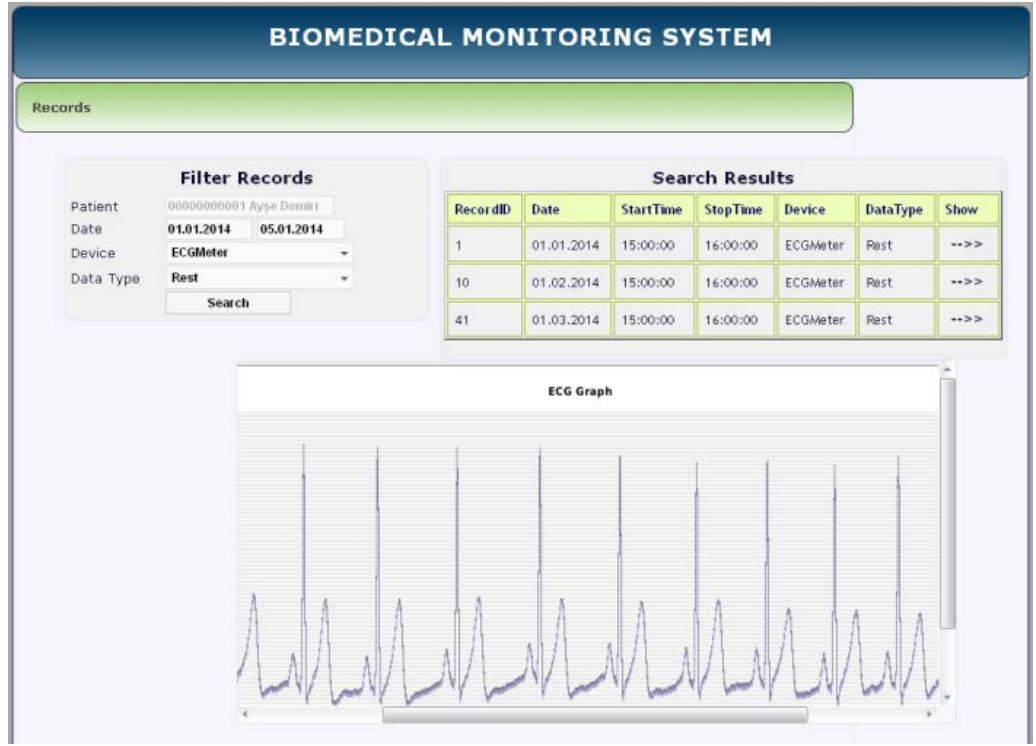

Figure 3. Web based user interface.

\section{Simulation Study and Discussion}

In order to investigate performance of the developed models and algorithms, a case study that includes sensing, transferring, storing and web based monitoring processes of ECG signal is evaluated using the framework. The case study illustrated in Figure 5 is modeled and simulated using OPNET Modeler as stated earlier. Database system (designed using MySQL) and Web based monitoring system (includes Apache web server, PHP, AJAX and HTML) are integrated into the Server node deployed on Figure 5. On the other hand, MATLAB interfaces are incorporated into the AP and Server nodes in the simulation model.

The sequence diagram of the simulation study is outlined in Figure 6. SN1 node senses the ECG signal from human body (SN1 reads the values from "ECG.txt" file in simulation runtime for more realistic performance 


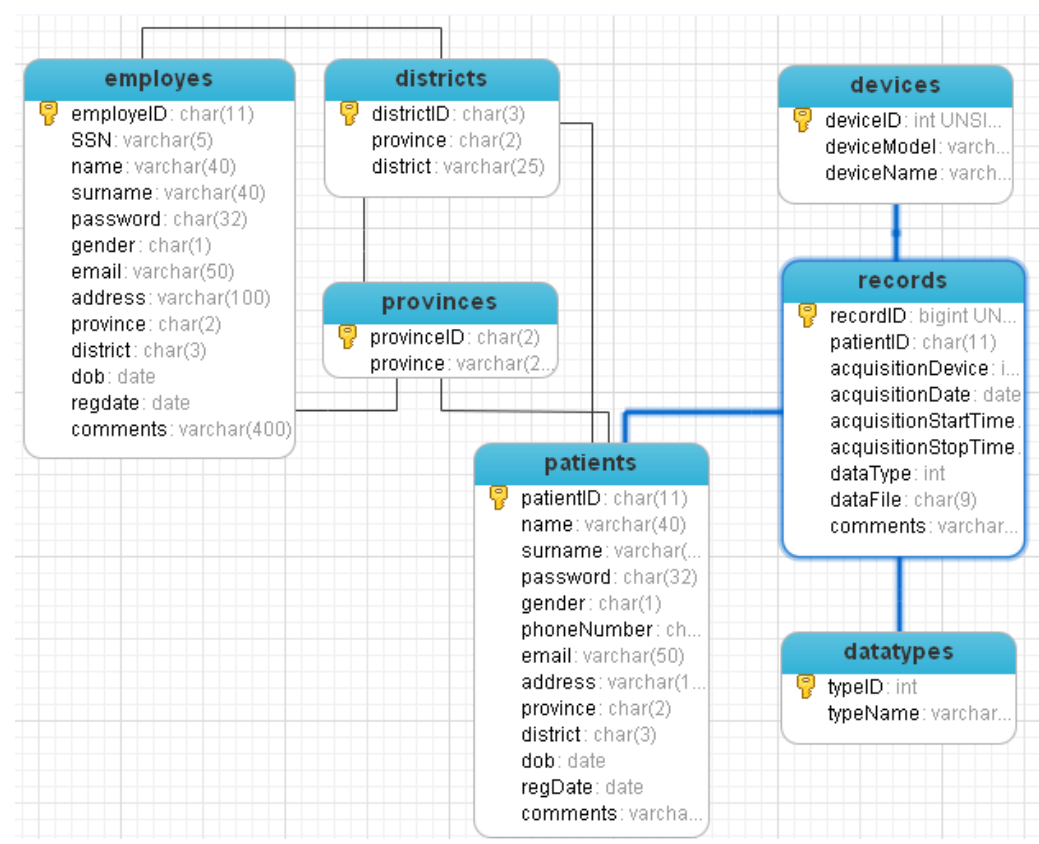

Figure 4. Relational model of the database designed.

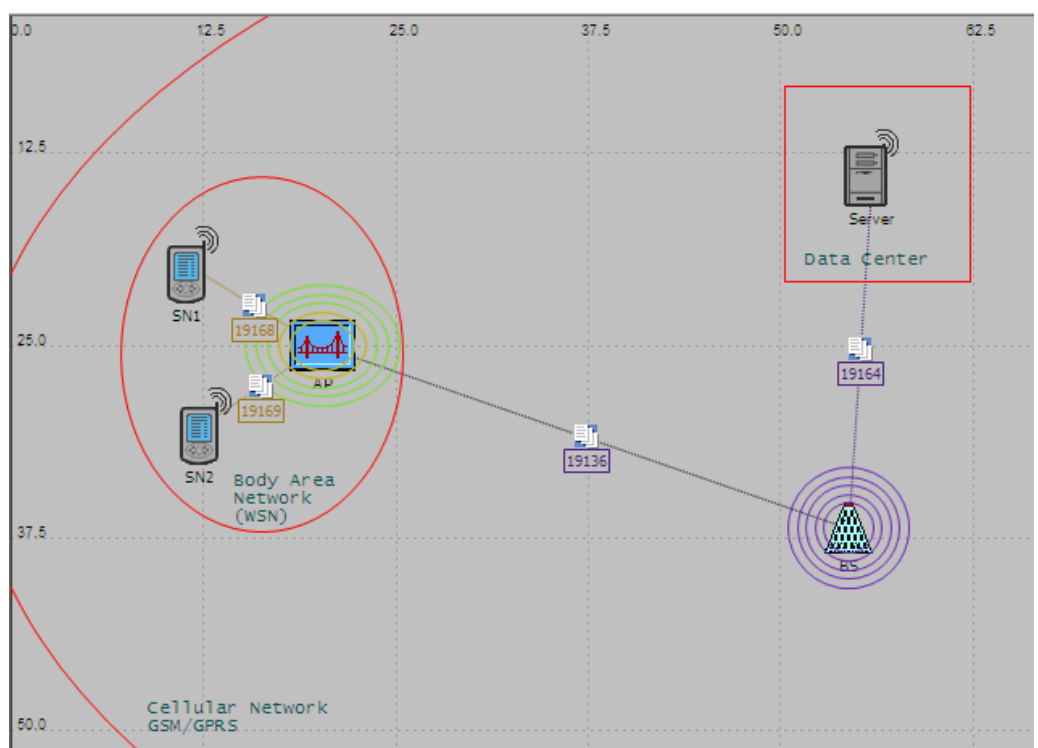

Figure 5. Simulation model.

results) and transmits to the AP using wireless ZigBee protocol. AP then delivers this data to the Server node in Data Center using GSM/GPRS technology. After the sensed biological data arrives at the Server, it is stored in the relational database designed (Figure 4) for further considerations. Finally, the registered employees can monitor the related records of any patient by the web based user interface developed (Figure 3).

In the light of discussions given above, it can be easily deduced that healthcare information systems are vital components for better coordination of healthcare and they have several components each is a big concern by itself. As stated formerly, the framework proposed here is considered to support potential upcoming studies. Some of them are summarized as follows:

- $\mathrm{AP}$ and Server nodes in the framework are able to communicate with MATLAB (The ECG graph given in Figure 7 is created with MATLAB during the simulation runtime) so that the rich set of MATLAB toolboxes can be employed in research studies related to decision support systems or preprocessing systems. 


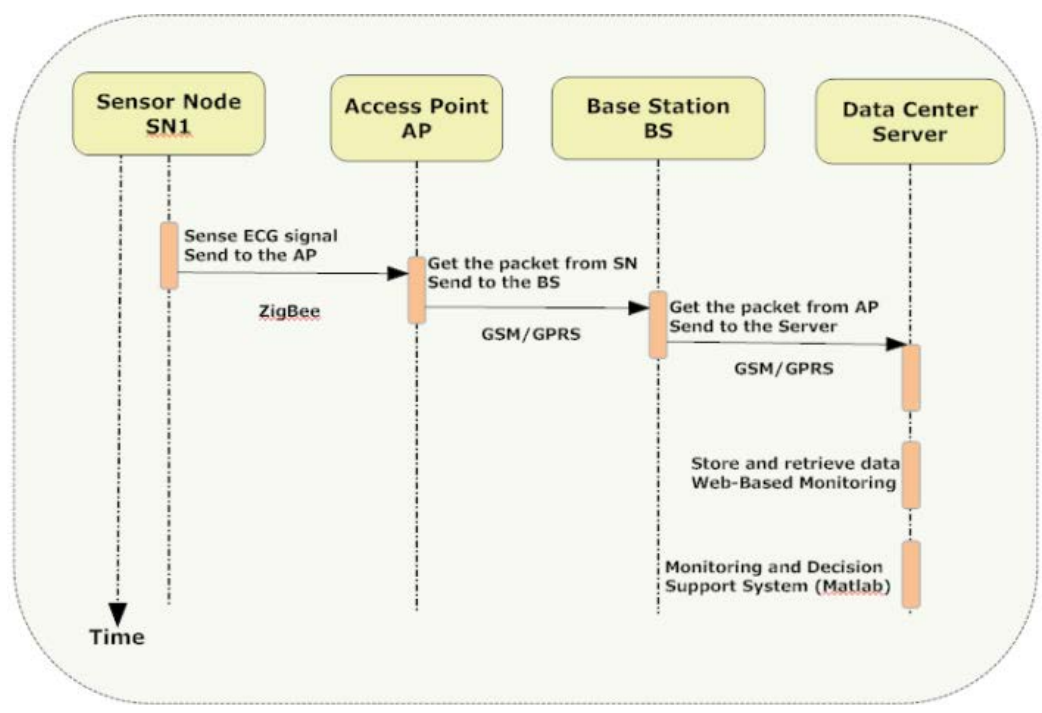

Figure 6. Sequence diagram of the simulation study.

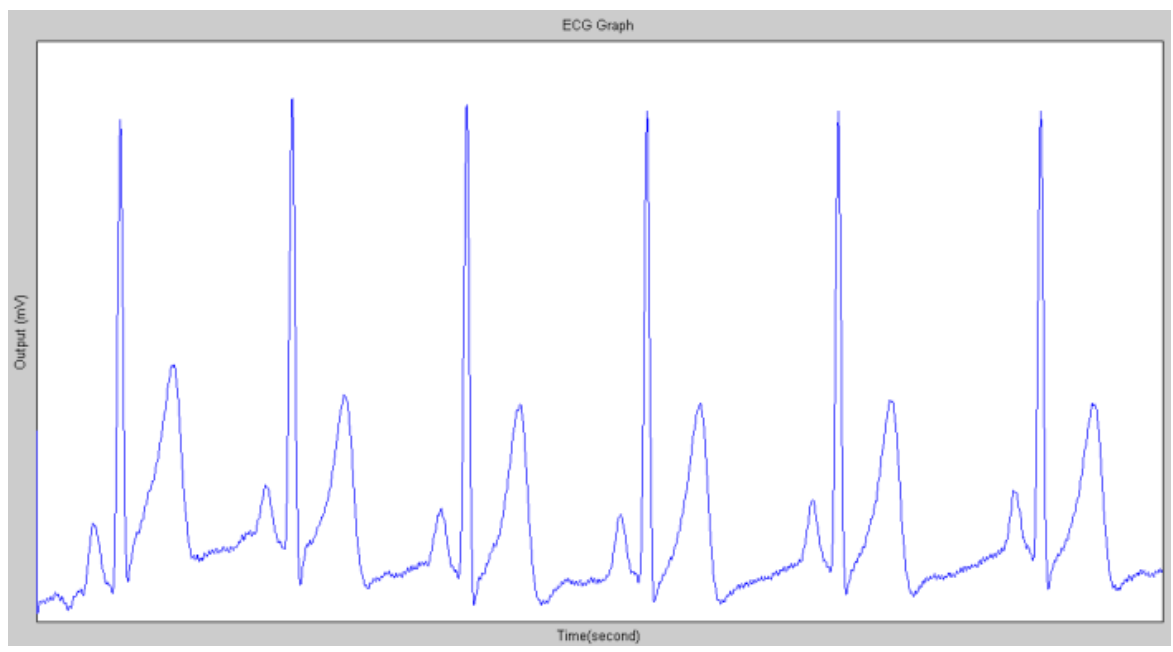

Figure 7. Example ECG signal graph created with MATLAB interfaces.

- AP and Server nodes in the framework can be provided with an alert and early warning subsystem for anomalous situations.

- A compression or future extraction subsystems can be incorporated into AP node so that the size of the transferred and stored data can be reduced for cost effective solutions.

- Instead of ZigBee and GSM/GPRS protocols, more efficient protocols can be studied, since OPNET Modeler supports most of the standard protocols and can allow development of custom protocols.

\section{Conclusion}

Healthcare information systems are crucial components for better coordination of healthcare and for good decision making. Since these systems have so many components and complex infrastructures, making research studies may be difficult in this area. The healthcare information system framework proposed here aimed at providing an infrastructure for the related research studies. In order to validate components of the framework developed, a case study that includes sensing, transferring, storing and web based monitoring processes of ECG signal is also realized. The results show that the framework presented here can be employed as a healthcare information system. It can also be concluded that, the framework developed can be used as an infrastructure in related research activities and consequently, lots of time can be saved from creating an experimental environment. 


\section{References}

[1] World Health Organization (2008) Health Metrics Network Framework and Standards for Country Health Information Systems. World Health Organization.

[2] World Health Organization (2008) Health Information Systems. World Health Organization.

[3] Vega, D.E., Schieferdecker, I. and Din, G. (2010) Design of a Test Framework for Automated Interoperability Testing of Healthcare Information Systems. 2nd International Conference on eHealth, Telemedicine, and Social Medicine, 2010. ETELEMED’10, 10-16 February 2010, 134-140.

[4] Vega, D.E. (2010) Towards an Automated and Dynamically Adaptable Test System for Testing Healthcare Information Systems. 3rd International Conference on Software Testing, Verification and Validation, 331-334. http://dx.doi.org/10.1109/ICST.2010.67

[5] Al-Shammary, D. and Khalil, I. (2010) A new XML-Aware Compression Technique for Improving Performance of Healthcare Information Systems over Hospital Networks. 32nd Annual International Conference on Engineering in Medicineand Biology Society (EMBC), 31 August-4 September 2010, 4440-4443.

[6] Kumar, C.S., Rao, C.V.G. and Govardhan, A. (2010) A Framework for Interoperable Healthcare Information Systems. International Conference on Computer Information Systems and Industrial Management Applications (CISIM), 8-10 October 2010, 604-608.

[7] Demirkan, H. (2013) A Smart Healthcare Systems Framework. IEEE IT Professional, 15, 38-45. http://dx.doi.org/10.1109/MITP.2013.35

[8] Trigo, J.D., et al. (2012) An Integrated Healthcare Information System for End-to-End Standardized Exchange and Homogeneous Management of Digital ECG Formats. IEEE Transactions on Information Technology in Biomedicine, 16, 518-529. http://dx.doi.org/10.1109/TITB.2012.2191296

[9] Zigbee Specifications, 2014. http://www.zigbee.org/Specifications/ZigBee/Overview.aspx 